Pacific Journal of Mathematics

NIVERSAL COSIMPLE IDOLS

March 1972 


\title{
UNIVERSAL COSIMPLE ISOLS
}

\author{
ERIK EllentuCK
}

Our results follow from a single priority scheme which we give in detail. They are (i) if an arbitrary $n+1$-ary relation over the nonnegative integers determines an $n$-ary function, then its canonical extension to the isols determines a function on the cosimple isols if and only if the function determined on the integers is an almost recursive combinatorial function, and (ii) every countable partially ordered set can be embedded in (a) the cosimple isols, and even more (b) the cosimple regressive isols. The remaining material generalizes and extends these results.

1. Independence. Let $\omega=$ the nonnegative integers, $P=$ the set of all subsets of $\omega$, and $Q=$ the set of all finite subsets of $\omega$. Use $X^{k} A$ for the $k$-fold direct power of $A$. The results of this section all follow from the priority method of [11]. Let $f: \omega \rightarrow X^{2} Q$ be a sequence of pairs $f(s)=\left(\alpha_{s}, \beta_{s}\right)$ where $\alpha_{s} \cap \beta_{s}=\varnothing$, and let $g: \omega \rightarrow \omega$. The requirement $R_{k}=\left\{\left(\alpha_{s}, \beta_{s}\right) \mid g(s)=k\right\} . \quad \xi \in P$ is said to meet the requirement $R_{k}$ if $\alpha \leqq \xi, \xi \cap \beta=\varnothing$ for some $(\alpha, \beta) \in R_{k}$. With every pair $(f, g)$ as above we associate the priority sequence $\xi: \omega \rightarrow Q$ constructed in stages. Stage $s=0, \xi_{0}=\varnothing$ and for stage $s>0, \xi_{s}=$ $\xi_{s-1}$ if (1), (2), or (3) below is true. Otherwise $\xi_{s}=\xi_{s-1} \cup \alpha_{s}$.

(1) $\xi_{s-1} \cap \beta_{s} \neq \varnothing$.

(2) there is an $r<s, r>0, g(r)<g(s)$ such that

$$
\alpha_{r} \varsubsetneqq \xi_{r-1}, \alpha_{r} \sqsubseteq \xi_{r}, \xi_{s-1} \cap \beta_{r}=\varnothing \text { and } \alpha_{s} \cap \beta_{r} \neq \varnothing \text {. }
$$

(3) there is an $r<s, r>0, g(r)=g(s)$ such that

$$
\alpha_{r} \nsubseteq \xi_{r-1}, \alpha_{r} \leqq \xi_{r} \text { and } \xi_{s-1} \cap \beta_{r}=\varnothing \text {. }
$$

The requirement $R_{k}$ is met at stage $s$ if $s>0, g(s)=k$ and $\alpha_{s} \nsubseteq \xi_{s-1}$ but $\alpha_{s} \subseteq \xi_{s}$. The requirement $R_{k}$ is injured at stage $s$ if for some $r<s, R_{k}$ was met at stage $r, \xi_{s-1} \cap \beta_{r}=\varnothing$ but $\xi_{s} \cap \beta_{r} \neq \varnothing$. The basic combinatorial content of the priority method is summarized in the fundamental

Lemma 1. (Sacks [11]). For each $k$ the set of stages $s$ where $R_{k}$ is either met or injured is finite.

Proof. First we need two facts, (4) and (5) below. They are

(4) if $R_{k}$ is injured at stage $s$ then for some $j<k, R_{j}$ is met at stage $s$. 
This follows because if $R_{k}$ is injured at stage $s$ then there is an $r<s$ such that $R_{k}$ was met at stage $r, \xi_{s-1} \cap \beta_{r}=\varnothing$ but $\xi_{s} \cap \beta_{r} \neq \varnothing$. Hence (1), (2), and (3) fail at stage $s$ and $r>0, g(r)=k, \alpha_{r} \nsubseteq \xi_{r-1}$ but $\alpha_{r} \subseteq \xi_{r}$. If $g(r)<g(s)$ then (2) is true at stage $s$ and if $g(r)=g(s)$ then (3) is true at stage $s$. Hence $g(s)<g(r)=k$. The second fact that we need is

(5) if $R_{k}$ is met at stages $r$ and $s$ where $r<s$ then for some $u, r<u<s$ and $R_{k}$ is injured at stage $u$.

This follows because if $R_{k}$ is met at stage $r$ then (1) fails at stage $r$, so $\xi_{r-1} \cap \beta_{r}=\varnothing$, and $\alpha_{r} \nsubseteq \xi_{r-1}$ but $\alpha_{r} \cong \xi_{r}$. Since $\alpha_{r} \cap \beta_{r}=\varnothing$ we have $\xi_{r} \cap \beta_{r}=\varnothing$ and since $R_{k}$ is met at stage $s$, (3) must fail at stage $s$. But $r>0$ and $g(r)=g(s)$ which implies $\xi_{s-1} \cap \beta_{r} \neq \varnothing$. Let $u$ be the least integer $r<u<s$ such that $\xi_{u} \cap \beta_{r} \neq \varnothing$. Clearly $R_{k}$ is injured at stage $u$.

We prove our lemma by induction. Suppose that for each $j<k$ the set of stages $s$ where $R_{j}$ is met or injured is finite. Choose $u<\omega$ such that for no $j<k$ and $s>u$ is $R_{j}$ met or injured at stage $s$. Then for $s>u, R_{k}$ cannot be injured at stage $s$ because otherwise by (4) $R_{j}$ would be met at stage $s$ for some $j<k$. Also for at most one $s>u$ is $R_{k}$ met at stage $s$ because otherwise by (5) $R_{k}$ would be injured at some stage $r>u$.

With every pair $(f, g)$ as above we associate the priority set $\xi=$ $\bigcup_{s<\omega} \xi_{s}$ where $\xi_{s}$ is the priority sequence associated with $(f, g)$. First note that if a requirement $R_{k}$ is met at stage $s$ and subsequently never injured then $\xi$ meets $R_{k}$. We lift recursive notions from $\omega$ to $Q$ via the canonical enumeration of finite sets and note that $\xi_{s}$ is recursive in the pair $(f, g)$ which implies that $\xi$ is r.e. in the pair $(f, g)$.

Let $j: X^{2} \omega \rightarrow \omega$ be the usual recursive one-to-one onto pairing function with first and second inverses $k, l$ respectively. Let $\omega^{n}=$ $\{j(x, n) \mid x<\omega\}$ and $\alpha^{n}=\alpha \cap \omega^{n} . \varphi_{n}(\gamma)$ is a partial recursive function which enumerates with index $n$ all partial recursive functions whose range and domain are contained in $Q$. Let $\left(n_{s}, \gamma_{s}, \delta_{s}\right)$ be a total recursive enumeration of $\left\{(n, \gamma, \delta) \mid \varphi_{n}(\gamma)=\delta\right\}$. We define $(f, g)$ as follows. $f(2 s+1)=\left(\alpha_{2 s+1}, \beta_{2 s+1}\right)$ where $\alpha_{2 s+1}=\delta_{s}-\gamma_{s}, \beta_{2 s+1}=\gamma_{s}$, and $g(2 s+$ $1)=2 n_{s}+1$. $f(2 s)=\left(\alpha_{2 s}, \beta_{2 s}\right)$ where $\alpha_{2 s}, \beta_{2 s}, \bigcup_{r<2 s}\left(\alpha_{r} \cup \beta_{r}\right)$ are pairwise disjoint, both $\alpha_{2 s}, \beta_{2 s}$ are subsets of $\omega^{k(k(s))}$ each containing $l(k(s))+1$ elements, and $g(2 s)=2 k(s)$. Let $\xi: \omega \rightarrow Q$ be the priority sequence associated with $(f, g)$ and let $\xi=\mathbf{U}_{s<\omega} \xi_{s}$ be the priority set associated with $(f, g)$. Throughout the remainder of this section $\xi_{s}, \xi$ will keep these meanings. Let $\eta=\omega-\xi$.

Lemma 2. $\eta^{m}$ is infinite for each $m<\omega$. 
Proof. Suppose $\eta^{m}$ contained $n$ elements. Let $k=2 j(m, n)$ and let $u<\omega$ be such that for no $s>u$ and $j \leqq k$ is $R_{j}$ either met or injured at stage $s$. Choose $s>u$ such that $g(s)=k$. Since $s>u$, $\xi_{s}=\xi_{s-1}$ and hence (1), (2), or (3) hold at stage $s$. By construction of $f$, both (1) and (2) fail at stage $s$, so (3) must hold. Hence there is an $r<s, r>0, g(r)=g(s)$ such that $\alpha_{r} \nsubseteq \xi_{r-1}, \alpha_{r} \leqq \xi_{r}$, and $\xi_{s-1} \cap \beta_{r}=\varnothing$. $\beta_{r}$ contains $n+1$ elements of $\omega^{m}$, and for $t \geqq s, \xi_{t} \cap \beta_{r}=\varnothing$ because otherwise $R_{k}$ would be injured at stage $t$. Hence $\beta_{r} \subseteq \eta$.

Let $M$ be the class of partial recursive functions $\varphi$ whose range and domain are both included in $Q$, which are increasing, i.e., if $\alpha \in$ domain $(\varphi)$ then $\alpha \subseteq \varphi(\alpha)$, and which are monotone, i.e., if $\alpha, \beta \in$ domain $(\varphi)$ and $\alpha \subseteq \beta$ then $\varphi(\alpha) \subseteq \varphi(\beta)$.

Lemma 3. For each $\varphi \in M$ there is a $\gamma \in Q, \gamma \subseteq \eta$ such that for all $\alpha \in Q, \gamma \subseteq \alpha \leqq \eta$ either $\alpha \notin$ domain $(\varphi)$ or $\varphi(\alpha)=\alpha$ or $\varphi(\alpha) \cap \xi \neq \varnothing$.

Proof. Let $\varphi$ have index $n$ in the enumeration of partial recursive functions above and let $k=2 n+1$. Choose $u<\omega$ such that for no $s>u$ and $j \leqq k$ is $R_{j}$ either met or injured at stage $s$. Choose $\gamma \in Q$ such that $\eta \cap \bigcup_{r \leqq u} \beta_{r} \varsubsetneqq \gamma \sqsubseteq \eta$. Suppose there were an $\alpha \in Q$, $\gamma \subseteq \alpha \subseteq \eta$ such that $\alpha \in$ domain $(\varphi)$ and $\alpha \varsubsetneqq \varphi(\alpha)$. Then for some $s$, $\alpha_{s}=\varphi(\alpha)-\alpha, \beta_{s}=\alpha$, and $g(s)=k$. $s>u$ for otherwise $\alpha \varsubsetneqq \gamma$. Hence $\xi_{s}=\xi_{s-1}$ so that (1), (2), or (3) must hold at stage $s$. Since $\xi_{s-1} \subseteq \xi$ and $\alpha \subseteq \eta$, (1) fails at stage $s$. If there were an $r<s, r>0$, $g(r)=j<k$ such that $R_{j}$ is met at stage $r$ and $\xi_{s-1} \cap \beta_{r}=\varnothing$ then $\beta_{r} \subseteq \eta$ since otherwise $R_{j}$ would be injured at some stage $t>u$. Hence $\beta_{r} \subseteq \alpha$ and so (2) fails at stage $s$. Hence (3) holds at stage $s$ and there is an $r<s, r>0, g(r)=g(s)=k$ such that $\alpha_{r} \nsubseteq \xi_{r-1}, \alpha_{r} \subseteq \xi_{r}$ and $\xi_{s-1} \cap \beta_{r}=\varnothing$. $\quad \beta_{r} \leqq \eta$ for otherwise $R_{k}$ would be injured at some stage $t>u$. $\varnothing \neq \alpha_{r}=\varphi\left(\beta_{r}\right)-\beta_{r} \subseteq \xi$ and by monotonicity we have $\varphi(\alpha) \cap \xi \neq \varnothing$.

Let $\Lambda=$ the isols and for $\alpha \in P$ let $\operatorname{Req}(\alpha)=$ the recursive equivalence type of $\alpha . \Lambda_{z}$ (the cosimple isols) = the set of those isols which can be represented as $\operatorname{Req}(\alpha)$ where $\omega-\alpha$ is r.e. and $\Lambda_{z}^{\infty}=\Lambda_{z}-\omega$, i.e., the infinite cosimple isols. For the rest of this section we fix the following notation $S=\left\{y_{m} \mid m<\omega\right\}$ where $y_{m}=\operatorname{Req}\left(\eta^{m}\right)$ for $m<\omega$.

THEOREM 1. $S$ is an infinite subset of $\Lambda_{z}^{\infty}$.

Proof. First we show that $\eta$ is immune. $\eta$ is infinite by Lemma 2. Suppose that $\eta$ contained an infinite r.e. subset $\beta$ which we assume is enumerated by a total recursive function $b(n)$. Define a recursive function $\varphi: Q \rightarrow Q$ by letting $\varphi(\alpha)=\alpha \cup\{b\}$ where $b$ is the first element 
in the enumeration $b(n)$ which does not occur in $\alpha$. Since $\varphi \in M$ there is a $\gamma \in Q, \gamma \subseteq \eta$ such that for all $\alpha \in Q, \gamma \subseteq \alpha \leqq \eta$ either $\alpha \notin$ domain $(\varphi)$ or $\varphi(\alpha)=\alpha$ or $\varphi(\alpha) \cap \xi \neq \varnothing$. Since all three of these possibilities are ruled out by our construction, $\eta$ is immune. $\xi$ is r.e. since it is r.e. in $(f, g)$, the latter clearly being recursive. Each $\xi^{m}$ is r.e. since it is the intersection of two r.e. sets and each $\eta^{m}$ is immune since it is an infinite subset of an immune set. Thus $S \subseteq \Lambda_{z}^{\infty}$. Next we show that if $m<n<\omega$ then $y_{m} \neq y_{n}$. Otherwise there would be a oneone partial recursive function $p$ which maps $\eta^{m}$ onto $\eta^{n}$. Define a recursive function $\varphi: Q \rightarrow Q$ by letting $\varphi(\alpha)=\alpha \cup p\left(\alpha^{m}\right)$. Since $\varphi \in M$ there is a $\gamma \in Q, \gamma \subseteq \eta$ which satisfies the conclusion of Lemma 3. By our construction $\varphi(\alpha)=\alpha$ for each $\alpha \in Q, \gamma \subseteq \alpha \leqq \eta$. Choose $x \in \eta^{m}-$ $p^{-1}(\gamma)$ so that $p(x) \in \varphi(\gamma \cup\{x\})=\gamma \cup\{x\}$. Since $p(x) \notin \gamma$ we have $p(x)=$ $x$ so that $\eta^{m} \cap \eta^{n} \neq \varnothing$, a contradiction.

A set $A$ of isols is called strongly recursively independent if for each $n>0, R \subseteq X^{n+1} \omega$ satisfying $\left(\forall v_{0}, \cdots, v_{n-1} \in \omega\right)\left(\exists ! v_{n} \in \omega\right)\left(v_{0}, \cdots\right.$, $\left.v_{n}\right) \in R$, and distinct elements $x_{0}, \cdots, x_{n-1} \in A$, if there is an $x_{n} \in \Lambda$ such that $\left(x_{0}, \cdots, x_{n}\right) \in R_{1}$ then $R$ is the graph of an eventually recursive combinatorial function.

\section{THEOREM 2. $S$ is strongly recursively independent.}

Proof. Let $n, R, x_{0}, \cdots, x_{n-1}$ be as in the hypothesis of the above definition. Using Theorem 3.1 of [10] $\left((R \times S)_{A}=R_{A} \times S_{A}\right.$, and $\omega_{A}=$ 1) there is no loss of generality in assuming that $x_{m}=y_{m}$ for $m<$ $n$. Suppose there is a $z \in \Lambda$ such that $\left(y_{0}, \cdots, y_{n-1}, z\right) \in R_{\Delta}$. For $\alpha \in$ $P$ let $\bar{\alpha}=\left(\alpha^{0}, \cdots \alpha^{n-1}\right)$. Then there is a $\zeta \in z$ and a recursive $R$-frame $F$ such that $(\bar{\eta}, \zeta) \in \mathscr{A}(F)$. If $\alpha=\left(\alpha_{0}, \cdots, \alpha_{n-1}\right)$, where possibly some of the $\alpha_{i}$ are themselves tuples, and $C_{F}(\alpha)$ is defined, define $C_{F}^{\vee}\left(\alpha_{0}, \cdots\right.$, $\left.\alpha_{i}^{v}, \cdots, \alpha_{n-1}\right)=\beta_{i}$ where $C_{F}(\alpha)=\left(\beta_{0}, \cdots, \beta_{n-1}\right)$ and $\alpha_{j} \leqq \beta_{j}$ for $j<n$ (here $\sqrt{ }$ occurs as a superscript to exactly one $\alpha_{i}$ and $\leqq$ denotes componentwise inclusion). Now define a partial recursive function $\varphi$ whose range and domain are included in $Q$ as follows. $\varphi(\alpha)=\alpha \cup \beta$ where $\bar{\beta}=C_{F}^{\vee}\left(\bar{\alpha}^{\vee}, \varnothing\right)$. Since $\varphi \in M$, Lemma 3 yields a $\gamma \in Q, \gamma \subseteq \eta$ such that for all $\alpha \in Q, \gamma \subseteq \alpha \subseteq \eta$, either $\alpha \notin$ domain $(\varphi)$ or $\varphi(\alpha)=\alpha$ or $\varphi(\alpha) \cap \xi \neq$ $\varnothing$. Since $(\bar{\eta}, \zeta) \in \mathscr{A}(F)$ only the second of these alternatives can occur, i.e., $\varphi(\alpha)=\alpha$. But this implies that $C_{F}(\alpha, \varnothing)=\left(\alpha, C_{F}^{\vee}\left(\alpha, \varnothing^{\prime}\right)\right)$ for each $\alpha \in A=\left\{\alpha \in X^{n} Q \mid \bar{\gamma} \leqq \alpha \leqq \bar{\eta}\right\}$. If $\alpha \in A$ let $\psi(\alpha)=C_{F}^{\vee}\left(\alpha, \varnothing^{\vee}\right)$ so that $(\alpha, \psi(\alpha)) \in F$. Let $\wedge$ denote componentwise intersection and observe that $\left(\alpha \wedge \alpha^{\prime}, \varnothing\right) \leqq(\alpha, \psi(\alpha)) \wedge\left(\alpha^{\prime}, \psi\left(\alpha^{\prime}\right)=\left(\alpha \wedge \alpha^{\prime}, \psi(\alpha) \cap \psi\left(\alpha^{\prime}\right)\right)\right.$ so that $\psi\left(\alpha \wedge \alpha^{\prime}\right) \subseteq \psi(\alpha) \cap \psi\left(\alpha^{\prime}\right)$. But then our hypothesis that $R$ is the graph of a function give (i) $\psi\left(\alpha \wedge \alpha^{\prime}\right)=\psi(\alpha) \cap \psi\left(\alpha^{\prime}\right)$ and (ii) $\alpha \sim \alpha^{\prime}$ componentwise implies $\psi(\alpha) \sim \psi\left(\alpha^{\prime}\right)$ (here $\sim$ denotes equal cardinality) 
for every $\alpha, \alpha^{\prime} \in A$. For $m<n$ let $p_{m}$ be a one-to-one function mapping $\omega$ onto $\eta^{m}-\gamma^{m}$. No recursive condition is placed on the $p_{m}$ 's. For $\alpha=\left(\alpha_{0}, \cdots, \alpha_{n-1}\right) \in X^{n} \omega$ let $p(\alpha)=\left(p_{0}\left(\alpha_{0}\right), \cdots, p_{n-1}\left(\alpha_{n-1}\right)\right)$ and define a function $\varphi: X^{n} Q \rightarrow Q$ by $\varphi(\alpha)=\psi(\bar{\gamma} \vee p(\alpha))$ where $\vee$ denotes componentwise union. $\psi$ inherits properties (i) and (ii) from $\psi$ and is therefore a combinatorial operator inducing a combinatorial function $r: X^{n} \omega \rightarrow \omega$ such that if $\gamma^{m}$ has $c_{m}$ elements then $\left(x_{0}+c_{0}, \cdots, x_{n-1}+c_{n-1}, r\left(x_{0}, \cdots\right.\right.$, $\left.\left.x_{n-1}\right)\right) \in R$ for every $\left(x_{0}, \cdots, x_{n-1}\right) \in X^{n} \omega$. Thus $R$ is the graph of an eventually combinatorial function. Let $B=\left\{(\alpha, \beta) \in\left(X^{n} Q\right) \times Q \mid \alpha \wedge\right.$ $\bar{\gamma}=\varnothing$ and $\left.C_{F}(\alpha \vee \bar{\gamma}, \varnothing)=(\alpha \vee \bar{\gamma}, \beta)\right\} . \quad B$ is r.e. and if we let $|\cdot|$ denote componentwise cardinality, $\widetilde{R}=\left\{x \in X^{n+1} \omega \mid(\exists \alpha)(x=|\alpha|\right.$ and $\alpha \in B)\}$ is an r.e. set which is the graph of $r$. Thus $R$ is the graph of an eventually recursive combinatorial function.

\section{Applications.}

CoRollary 1. Let $n>0$ and $R \subseteq X^{n+1} \omega$ the graph of a function $r$. Then (i) $r$ is eventually recursive combinatorial if and only if for each $x \in X^{n} \Lambda_{z}^{\infty}$ there is exactly one $y \in \Lambda$ such that $(x, y) \in R_{A}$, (ii) $r$ is almost recursive combinatorial if and only if for each $x \in X^{n} \Lambda_{z}$ there is exactly one $y \in \Lambda$ such that $(x, y) \in R_{A^{*}}$. This solves the problem left open on the middle of page 247 of [7].

A set $A$ of isols is called recursively independent if for each $n>$ $0, R \subseteq X^{n} \omega$, and distinct elements $x_{0}, \cdots, x_{n-1} \in A$, if $\left(x_{0}, \cdots, x_{n-1}\right) \in$ $R_{\Delta}$ then there exists $\alpha \in Q$ such that $X^{n}(\omega-\alpha) \subseteq R$.

THEOREM 3. If $A$ is a strongly recursively independent set of isols, then $A$ is recursively independent.

Proof. For $R \cong X^{n} \omega$ let $\widetilde{R}=\{(x, 0) \mid x \in R\} \cup\left\{(x, 1) \mid x \in X^{n} \omega-R\right\}$. $\widetilde{R}$ is the graph of the characteristic function $r$ of $R$ and satisfies $(\forall x)(x \in R \rightarrow(x, 0) \in \widetilde{R})$ in $\omega$ and hence in $\Lambda$. Let $x_{0}, \cdots, x_{n-1}$ be distinct elements of $A$ and suppose that $\left(x_{0}, \cdots, x_{n-1}\right) \in R_{A}$. Then $\left(x_{0}, \cdots, x_{n-1}\right.$, $0) \in \widetilde{R}_{A}$ which implies that $r$ is eventually combinatorial. Since eventually combinatorial functions are eventually monotone, $r$ is eventually equal to 0 or eventually equal to 1 . If $r$ is eventually equal to 1 there is an $a<\omega$ such that $\left(\forall x_{0}, \cdots, x_{n-1}\right)\left(x_{0}+a, \cdots, x_{n-1}+a\right) \notin R$ holds in $\omega$ and hence in $\Lambda$. This implies that at least one of the $x_{i}$ 's in $A$ is finite, in fact <a. Clearly no member of a strongly recursively independent set can be finite so $r$ must be eventually equal to 0 .

CoRollaRY 2. $S$ is recursively independent. 
A set $A$ of isols is called independent if for each $n>1$ and distinct elements $x_{0}, \cdots, x_{n-1} \in A, x_{0} \not x_{1}+\cdots+x_{n-1}$.

THEOREM 4. If $A$ is a recursively independent set of isols then $A$ is independent.

Proof. Fix $n$ and let $R=\left\{\left(x_{0}, \cdots, x_{n-1}\right) \in X^{n} \omega \mid x_{0} \leqq x_{1}+\cdots+\right.$ $\left.x_{n-1}\right\}$. Then $\left(\forall x_{0}, \cdots, x_{n-1}, y\right)\left(x_{0}+y=x_{1}+\cdots+x_{n-1} \rightarrow\left(x_{0}, \cdots, x_{n-1}\right) \epsilon\right.$ $R$ ) holds in $\omega$ and hence in $\Lambda$. Thus if $x_{0}, \cdots, x_{n-1}$ are distinct elements of $A$ and $x_{0} \leqq x_{1}+\cdots+x_{n-1}$ then $\left(x_{0}, \cdots, x_{n-1}\right) \in R_{A}$, a contradiction.

\section{Corollary 3. $S$ is independent.}

Let $A$ be a set partially ordered by a relation $R$. We say that $\langle A, R\rangle$ can be embedded in $\Lambda$ if there exists an order isomorphism $h: A \rightarrow \Lambda$ with respect to the usual ordering of $\Lambda$. The following theorem is of interest when compared with results in [5].

THEOREM 5. Every countable partially ordered set can be embedded in the cosimple isols.

Proof. By a result of [9] there is a recursive relation $R$ which partially orders $\omega$ in such a way that every countable partial ordering can be embedded in $\langle\omega, R\rangle$. We prove our theorem by embedding $\langle\omega, R\rangle$ in $\Lambda_{z}$. Assume that $R$ is reflexive and define $h(n)=\cup\left\{\eta^{m} \mid m R n\right\}$. $h(n)$ is an immune set whose complement in $\omega$ is $U\left\{\omega^{m} \mid \sim m R n\right\} \cup$ $\left(\xi \cap \cup\left\{\omega^{m} \mid m R n\right\}\right)$ which clearly is an r.e. set. Thus Req $(h(n)) \in \Lambda_{z}$. If $n_{0} R n_{1}$ then $h\left(n_{0}\right) \subseteq h\left(n_{1}\right)$ and $h\left(n_{0}\right), h\left(n_{1}\right)-h\left(n_{0}\right)$ are recursively separated by the recursive set $\cup\left\{\omega^{m} \mid m R n_{0}\right\}$. Thus Req $\left(h\left(n_{0}\right)\right) \leqq$ $\operatorname{Req}\left(h\left(n_{1}\right)\right)$ by the identity function. If $\sim m R n$ but Req $(h(m)) \leqq$ Req $(h(n))$ then for some one-one partial recursive function $p, \eta^{m} \subseteq$ domain $(p)$ and $p$ maps $\eta^{m}$ into $\cup\left\{\eta^{j} \mid j \neq m\right\}$. Define a partial recursive function $\phi$ whose range and domain are included in $Q$ by $\varphi(\alpha)=$ $\alpha \cup p\left(\alpha^{m}\right)$. Then $\varphi(\alpha)=\alpha$ for all $\alpha \in Q, \gamma \subseteq \alpha \subseteq \eta$ where $\gamma$ is given by Lemma 3. If $x \in \eta^{m}-p^{-1}(\gamma)$ then $p(x) \in \varphi(\gamma \cup\{x\})=\gamma \cup\{x\}$. Since $p(x) \notin \gamma$ we have $p(x)=x$ so $\eta^{m} \cap \cup\left\{\eta^{j} \mid j \neq m\right\} \neq \varnothing$, a contradiction. Thus Req $(h(m)) \geqq$ Req $(h(n))$ and we see that Req $(h(n))$ is the required order isomorphism.

There are several ways to extend Theorem 5 . If $R^{\prime}$ is a partial ordering of $\Lambda$ say that $\langle A, R\rangle$ is $R^{\prime}$-embedded in $\Lambda$ if the mapping $h: A \rightarrow \Lambda$ is an $R-R^{\prime}$ order isomorphism. For $R^{\prime}$ we are interested in $<_{\Lambda}$, the canonical extension to $\Lambda$ of $R=\left\{(x, y) \in X^{2} \omega \mid x<y\right\}$, and $\leqq_{1}$, 
the canonical extension to $A$ of $R=\left\{(x, y) \in X^{2} \omega \mid x \leqq y\right\}$. Then we have

COROLLARY 4. Every countable partially ordered set can be both $<_{A}$ and $\leqq_{A}$ embedded in $\Lambda_{z}$.

Proof. Let $R, h$ be as in the proof of Theorem 5. First we show that if $m R n$ and $m \neq n$ then Req $(h(m))<_{A} \operatorname{Req}(h(n))$. Since $m R n$ and $m \neq n, h(m) \varsubsetneqq h(n)$. Let $x \in h(n)-h(m)$ and let $F=\left\{(\alpha, \beta) \in X^{2} Q \mid x \notin \alpha\right.$ and $\alpha \cup\{x\} \subseteq \beta\} . \quad F$ is a recursive <-frame from which $(h(m), h(n))$ is attainable. Next we show that if $\sim m R n$ then $\sim \operatorname{Req}(h(m)) \leqq_{A} \operatorname{Req}(h(n))$. For suppose that $F$ is a recursive $\leqq$-frame and $(h(m), h(n)) \in \mathscr{A}(F)$. Since $\sim m R n, \eta^{m} \subseteq h(m)$ and $\eta^{m} \cap h(n)=\varnothing$. Now define a partial recursive function $\varphi$ whose range and domain are included in $Q$ as follows. $\varphi(\alpha)=\alpha \cup \beta$ where $\beta=\beta_{0} \cup \beta_{1}$ and $C_{F}\left(\alpha^{m}, \varnothing\right)=\left(\beta_{0}, \beta_{1}\right)$. Since $\varphi \in M$ Lemma 3 gives the required $\gamma$. If $\alpha \subseteq \eta^{m}$ then $\varphi(\alpha \cup \gamma)=\alpha \cup \gamma$ which implies that if $C_{F}\left(\alpha^{m} \cup \gamma^{m}, \varnothing\right)=\left(\beta_{0}, \beta_{1}\right)$ then $\beta_{1} \leqq \gamma$. Since $\alpha \subseteq \eta^{m}$ can be made as large as we please, $F$ is not a $\leqq$-frame. Hence $(h(m), h(n)) \notin$ $\mathscr{A}(F)$. To complete our proof recall that for $u, v \in \Lambda, u<v \rightarrow u<_{\Lambda} v \rightarrow$ $u \leqq_{4} v, u \leqq v \rightarrow u \leqq_{1} v$, and $u=v \rightarrow u \leqq_{1} v$, all implied by a metatheorem.

Another way to extend Theorem 5 is to try to embed partially ordered sets into the cosimple regressive isols $\Lambda_{r z}$. Let $\tau$ be an infinite retraceable set enumerated in increasing order as $\left\{t_{n} \mid n<\omega\right\}$ where $t_{n}<t_{n+1}$ for $n<\omega$ and let $p$ be a partial recursive special (cf. [3]) retracing function for $\tau$. $\tau$ is called T-retraceable if for every partial recursive function $h$ there is $u<\omega$ such that for all $n>u$ either $t_{n} \notin$ domain $(h)$ or $h\left(t_{n}\right)<t_{n+1}$. By a result of [8] T-retraceable sets with r.e. complement exist and they are necessarily immune. Fix $\tau$ in the following discussion as $T$-retraceable with r.e. complement, $p$ is a total recursive function (cf. P.5 of [3]) and $p^{*}(x)=(\mu n)\left(p^{n}(x)=\right.$ $\left.p^{n+1}(x)\right)$.

LemMa 4. If $\alpha, \beta \subseteq \tau$ are infinite recursively equivalent sets then $\alpha \cap \beta \neq \varnothing$.

Proof. Let $h$ be a partial recursive one-to-one function mapping $\alpha$ onto $\beta$. By $T$-retraceability there is a $u<\omega$ such that for all $n>$ $u$, if $t_{n} \in \alpha$ then $h\left(t_{n}\right) \leqq t_{n}$ (since $h\left(t_{n}\right)<t_{n+1}$ and $h\left(t_{n}\right) \in \beta \leqq \tau$ ), and if $t_{n} \in \beta$ then $h^{-1}\left(t_{n}\right) \leqq t_{n}$. Recast the second conclusion as $t_{n} \leqq h\left(t_{n}\right)$ if $h\left(t_{n}\right) \in \beta$ and $p^{*}\left(h\left(t_{n}\right)\right)>u$. Choose $u^{\prime}>u$ such that $p^{*}\left(h\left(t_{n}\right)\right)>u$ for 
$n>u^{\prime}$. Thus if $n>u^{\prime}$ and $t_{n} \in \alpha$ then $h\left(t_{n}\right) \leqq t_{n} \leqq h\left(t_{n}\right)$, i.e., $t_{n} \in \alpha \cap \beta$.

THEOREM 6. Every countable partially ordered set can be embedded in the cosimple regressive isols.

Proof. Let $R$ be as in the proof of Theorem 5, $\sigma=\omega-\tau$, and for $m<\omega$ let $\mu^{m}=\left\{x<\omega \mid k\left(p^{*}(x)\right)=m\right\}$. For any set $\alpha$ let $\alpha^{m}=$ $\alpha \cap \mu^{m}$. Our embedding is defined by $h(n)=\cup\left\{\tau^{m} \mid m R n\right\}$. To show that $h(n)$ is retraceable note that $h(n)$ is recursively separated from $\tau-h(n)$ by the recursive set $\cup\left\{\mu^{m} \mid m R n\right\}$ and then use P.5 of [2] to conclude that $h(n)$ is retraceable. $h(n)$ is an immune set whose complement in $\omega$ is $\cup\left\{\mu^{m} \mid \sim m R n\right\} \cup\left(\sigma \cap \cup\left\{\mu^{m} \mid m R n\right\}\right)$ which is clearly r.e. Thus Req $(h(n)) \in \Lambda_{r z}$. If $n_{0} R n_{1}$ then $h\left(n_{0}\right) \subseteq h\left(n_{1}\right)$ and $h\left(n_{0}\right), h\left(n_{1}\right)-$ $h\left(n_{0}\right)$ are recursively separated by the recursive set $\cup\left\{\mu^{m} \mid m R n_{0}\right\}$. Therefore $\operatorname{Req}\left(h\left(n_{0}\right)\right) \leqq \operatorname{Req}\left(h\left(n_{1}\right)\right)$. If $\sim m R n$ but $\operatorname{Req}(h(m)) \leqq \operatorname{Req}(h(n))$ then for some one-to-one partial recursive function $q, \tau^{m} \subseteq$ domain $(q)$ and $q$ maps $\tau^{m}$ into $\cup\left\{\tau^{j} \mid j \neq m\right\}$. Since this is ruled out by Lemma 4 Req $(h(n))$ is the required isomorphism.

Let $S_{r}=\left\{z_{m} \mid m<\omega\right\}$ where $z_{m}=\operatorname{Req}\left(\tau^{m}\right)$ for $m<\omega$.

CoRollary 5. $S_{r}$ is an infinite independent but not recursively independent subset of $\Lambda_{r r}^{\infty}$.

Proof. We only show that $S_{r}$ is not recursively independent. The rest is obvious from the proof of Theorem 6. If $n>m$ then $j(n, x)>j(m, x)$. Define a function $q$ on $\mu^{n}$ by $q(x)=(\mu y)\left(\exists s \leqq p^{*}(x)\right)$ $\left(y=p^{s}(x)\right.$ and $\left.p^{*}(y)=j\left(m, l\left(p^{*}(x)\right)\right)\right) . q$ is readily seen to be a many one partial recursive function which maps $\tau^{n}$ one-to-one onto $\tau^{m}$. Hence $\operatorname{Req}\left(\tau^{n}\right) \leqq * \operatorname{Req}\left(\tau^{m}\right)$ and therefore $\operatorname{Req}\left(\tau^{n}\right) \leqq{ }_{1} \operatorname{Req}\left(\tau^{m}\right)$ by Theorem 2.1 of [1] (which asserts that $\varliminf^{*}$ agrees with $\leqq_{\Lambda}$ on $\Lambda_{r}$.

Corollary 5 explains why in Theorem 6 our method did not give $\leqq_{A}$ or $<_{A}$ embeddings. In [6] it is shown that Req $(\tau)$ where $\tau$ is $T$-retraceable is universal, i.e., $\{\operatorname{Req}(\tau)\}$ is recursively independent. Call $x \in \Lambda$ strongly universal if for each $R \cong X^{2} \omega$ satisfying $\left(\forall v_{0}\right)\left(\exists ! v_{1}\right)\left(v_{0}, v_{1}\right) \in R$, if there is a $y \in \Lambda$ such that $(x, y) \in R_{A}$ then $R$ is the graph of an eventually recursive increasing function.

THEOREM 7. Req $(\tau)$ is strongly universal.

Proof. Let $R$ be as in the hypothesis of the above definition. Assume there is an isolated $\zeta \leqq \omega$ and recursive $R$-frame $F$ such that $(\tau, \zeta)$ is attainable from $F$. For $x<\omega$ let $s(x)=\left\{p^{n}(x) \mid n \leqq\right.$ 
$\left.p^{*}(x)\right\}$ and let $\gamma=\left\{x<\omega \mid(s(x), \varnothing) \in F^{*}\right\}$. Clearly $s(x)$ is a recursive function and $\gamma$ is an r.e. set. For $x \in \gamma$ let $h(x)=$ the maximum element of $C_{F}^{\vee}\left(s(x)^{\nu}, \varnothing\right) . \quad h(x)$ is partial recursive and since $\tau \leqq \gamma$, defined on all of $\tau$ and maps $\tau$ into $\tau$. Hence there is a $u<\omega$ such that $h\left(t_{n}\right) \leqq$ $t_{n}$ for $n>u$. This implies that $C_{F}^{\vee}\left(s(x)^{\vee}, \varnothing\right)=s(x)$ for all $x \in \tau$ with $p^{*}(x)>u$, and consequently for such $x$ we have $C_{F}(s(x), \varnothing)=(s(x)$, $\left.C_{F}^{\vee}\left(s(x), \varnothing^{\vee}\right)\right) \in F$. Clearly the set $\left\{(m, n) \mid(\exists x)\left(x \in \tau\right.\right.$ and $p^{*}(x)>u$ and $m=|s(x)|$ and $\left.\left.n=\left|C_{F}^{\vee}\left(s(x), \varnothing^{\vee}\right)\right|\right)\right\}$ is the graph of an increasing function contained in $R$ and has a recursive extension $\{(m, n)\{(\exists \alpha, \beta)(\alpha$, $\beta) \in F$ and $m=|\alpha|$ and $n=|\beta|\}$ so $R$ itself is the graph of an eventually recursive increasing function.

\section{CoRollary 6. Req $(\tau)$ is universal.}

Proof. Just as in the proof of Theorem 3 using the fact that a bounded eventually increasing function is eventually constant. q.e.d.

Using a somewhat different priority method, we have been able to extend the results of this paper to the cosimple regressive isols. They are stated below without proof for comparison with Corollary 1. If $f: \omega \rightarrow \omega$ let $\Delta f(x)=f(x+1)-f(x)$. If $f: X^{n} \omega \rightarrow \omega$ and $i<n$ let $\Delta_{i}$ be just like $\Delta$ except that it is applied to the $i$ th argument of $f$. Let $\Delta=$ the composition $\Delta_{0} \cdots \Delta_{n-1}$. Then $\Delta f$ is well defined and is consistent with the notation for the one variable case. $f$ is called recursive increasing if $f$ is recursive and $\Delta \hat{f}(x) \geqq 0$ for all $x \in X^{n} \omega$. where $\hat{f}\left(x_{0}+1, \cdots, x_{n-1}+1\right)=f\left(x_{0}, \cdots, x_{n-1}\right)$ and $\hat{f}(x)=0$ otherwise. Eventually recursive increasing and almost recursive increasing are canonically defined.

THEOREM 8. Let $n>0$ and $R \cong X^{n+1} \omega$ the graph of a function $r$. Then $r$ is almost recursive increasing if and only if for each $x \in X^{n} \Lambda_{r z}$ there is a $y \in \Lambda$ such that $(x, y) \in R_{\Lambda}$.

A function $f: X^{n} \omega \rightarrow \omega$ is called recursive regular if there are unary recursive increasing functions $g_{i}$ for $i<n$ such that $f\left(x_{0}, \cdots, x_{n-1}\right)=$ minimum $\left\{g_{0}\left(x_{0}\right), \cdots, g_{n-1}\left(x_{n-1}\right)\right\}$. Eventually recursive regular and almost recursive regular are canonically defined.

Theorem 9. Let $n>0$ and $R \subseteq X^{n+1} \omega$ the graph of a function $r$. Then $r$ is almost recursive regular if and only if for each $x \in X^{n} \Lambda_{r z}$ there is a $y \in \Lambda_{r}$ such that $(x, y) \in R_{\Delta}$.

In both of these theorems the $y$ in question, when it exists, is actually an element of $\Lambda_{z}$. 


\section{REFERENCES}

1. J. Barback, Two notes on recursive functions and regressive isols, TAMS, 144 (1969), 77-94.

2. J. C. E. Dekker, The minimum of two regressive isols, Math. Zeitschr., 83 (1964), $345-366$.

3. J. C. E. Dekker and J. R. Myhill, Retraceable sets, Canad. J. Math., 10 (1958), 357-373.

4. E. Ellentuck, On the form of fnnctions which preserve regressive isols, submitted to Composito Math.

5. Uncountable suborderings of the isols, submitted to Composito Math.

6. J. Gersting, A rate of growth criterion for universality of regressive isols, Pacific

J. Math., 31 (1969), 669-677.

7. L. Hay, The cosimple isols, Ann. of Math., 83 (1966), 231-256.

8. T. G. McLaughlin, Hereditarily retraceable isols, Bull. Amer. Math. Soc., 73 (1967), 113-115.

9. A. Mostowski, Über gewisse universelle Relationen, Ann. Soc. Polon. Math., 17 (1938), 117-118.

10. A. Nerode, Extensions to isols, Ann. of Math., 73 (1961), 362-403.

11. G. E. Sacks, Degrees of Unsolvability, Ann. of Math. Studies 55 (1963), Princeton.

Received April 27, 1971. Research supported in part by NSF contract GP-11509.

RUTGERS UNIVERSITY 


\section{PACIFIC JOURNAL OF MATHEMATICS}

\section{EDITORS}

\section{H. SAMELSON}

Stanford University

Stanford, California 94305

\section{R. HOBBY}

University of Washington

Seattle, Washington 98105

\section{J. DugundjI}

Department of Mathematics University of Southern California Los Angeles, California 90007

RICHARD ARENS

University of California

Los Angeles, California 90024

\section{ASSOCIATE EDITORS}
E. F. BECKENBACH
B. H. NeumanN
F. WOLF
K. YOSHIDA

\section{SUPPORTING INSTITUTIONS}

\author{
UNIVERSITY OF BRITISH COLUMBIA \\ CALIFORNIA INSTITUTE OF TECHNOLOGY \\ UNIVERSITY OF CALIFORNIA \\ MONTANA STATE UNIVERSITY \\ UNIVERSITY OF NEVADA \\ NEW MEXICO STATE UNIVERSITY \\ OREGON STATE UNIVERSITY \\ UNIVERSITY OF OREGON \\ OSAKA UNIVERSITY
}

\author{
UNIVERSITY OF SOUTHERN CALIFORNIA \\ STANFORD UNIVERSITY \\ UNIVERSITY OF TOKYO \\ UNIVERSITY OF UTAH \\ WASHINGTON STATE UNIVERSITY \\ UNIVERSITY OF WASHINGTON \\ AMERICAN MATHEMATICAL SOCIETY \\ NAVAL WEAPONS CENTER
}

The Supporting Institutions listed above contribute to the cost of publication of this Journal, but they are not owners or publishers and have no responsibility for its content or policies.

Mathematical papers intended for publication in the Pacific Journal of Mathematics should be in typed form or offset-reproduced, (not dittoed), double spaced with large margins. Underline Greek letters in red, German in green, and script in blue. The first paragraph or two must be capable of being used separately as a synopsis of the entire paper. The editorial "we" must not be used in the synopsis, and items of the bibliography should not be cited there unless absolutely necessary, in which case they must be identified by author and Journal, rather than by item number. Manuscripts, in duplicate if possible, may be sent to any one of the four editors. Please classify according to the scheme of Math. Rev. Index to Vol. 39. All other communications to the editors should be addressed to the managing editor, Richard Arens, University of California, Los Angeles, California, 90024.

50 reprints are provided free for each article; additional copies may be obtained at cost in multiples of 50 .

The Pacific Journal of Mathematics is published monthly. Effective with Volume 16 the price per volume (3 numbers) is $\$ 8.00$; single issues, $\$ 3.00$. Special price for current issues to individual faculty members of supporting institutions and to individual members of the American Mathematical Society: $\$ 4.00$ per volume; single issues $\$ 1.50$. Back numbers are available.

Subscriptions, orders for back numbers, and changes of address should be sent to Pacific Journal of Mathematics, 103 Highland Boulevard, Berkeley, California, 94708.

PUBLISHED BY PACIFIC JOURNAL OF MATHEMATICS, A NON-PROFIT CORPORATION

Printed at Kokusai Bunken Insatsusha (International Academic Printing Co., Ltd.), 270, 3-chome Totsuka-cho, Shinjuku-ku, Tokyo 160, Japan. 


\section{Pacific Journal of Mathematics}

\section{Vol. 42, No. $3 \quad$ March, 1972}

Catherine Bandle, Extensions of an inequality by Pólya and Schiffer for vibrating membranes ................................ 543

S. J. Bernau, Topologies on structure spaces of lattice groups.......... 557

Woodrow Wilson Bledsoe and Charles Edward Wilks, On Borel product measures .......................................

Eggert Briem and Murali Rao, Normpreserving extensions in subspaces of

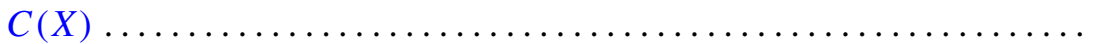

Alan Seymour Cover, Generalized continuation.................. 589

Larry Jean Cummings, Transformations of symmetric tensors .......... 603

Peter Michael Curran, Cohomology of finitely presented groups .......... 615

James B. Derr and N. P. Mukherjee, Generalized quasicenter and

hyperquasicenter of a finite group ...................... 621

Erik Maurice Ellentuck, Universal cosimple isols .................. 629

Benny Dan Evans, Boundary respecting maps of 3-mainfolds .......... 639

David F. Fraser, A probabilistic method for the rate of convergence to the

Dirichlet problem .................................. 657

Raymond Taylor Hoobler, Cohomology in the finite topology and Brauer

groups ..................................... 667

Louis Roberts Hunt, Locally holomorphic sets and the Levi form ........ 681

B. T. Y. Kwee, On absolute de la Vallée Poussin summability............ 689

Gérard Lallement, On nilpotency and residual finiteness in semigroups .... 693

George Edward Lang, Evaluation subgroups of factor spaces........... 701

Andy R. Magid, A separably closed ring with nonzero torsion pic ....... 711

Billy E. Rhoades, Commutants of some Hausdorff matrices ............. 715

Maxwell Alexander Rosenlicht, Canonical forms for local derivations . . . . 721

Cedric Felix Schubert, On a conjecture of L. B. Page ................ 733

Reinhard Schultz, Composition constructions on diffeomorphisms of $S^{p} \times S^{q}$

J. P. Singhal and H. M. (Hari Mohan) Srivastava, A class of bilateral generating functions for certain classical polynomials ....

Richard Alan Slocum, Using brick partitionings to establish conditions which insure that a Peano continuum is a 2-cell, a 2-sphere or an annulus...

James F. Smith, The p-classes of an $H^{*}$-algebra ...

Jack Williamson, Meromorphic functions with negative zeros and positive

poles and a theorem of Teichmuller ................. 